\title{
El conocimiento pedagógico del contenido (CPC) por el profesor universitario en el uso del método de resolución de problemas
}

\section{Pedagogical content knowledge (CPC) by the university professor in the use of the} problem solving method

\author{
Karen Patricia Agudelo Arteaga ${ }^{1}$ \\ Elvira Patricia Flórez Nisperuza² \\ Eduardo Ravanal Moreno ${ }^{3}$ \\ Roberto figueroa Molina ${ }^{4}$
}

\section{RESUMEN}

El estudio propone la caracterización del Conocimiento Pedagógico del Contenido, declarado por el profesor universitario de

$1 \quad \operatorname{Doctor}(C)$ en ciencias de la educación. Universidad de Córdoba- Colombia. Correo electrónico: karenagudelo@correo.unicordoba.edu.co . Orcid: https://orcid.org/00000003-0792-1155

2 Doctor en Ciencias de la Educación. Universidad de Córdoba-Colombia. Correo electrónico: epatriciaflorez@ correo.unicordoba.edu.co. Orcid: https://orcid.org/00000003-4621-8382

3 Doctor en educación. Universidad Bernardo O'Higgins-Santiago de Chile. Correo electrónico: eravanalmoreno@gmail.com. Orcid: https://orcid.org/00000003-2763-9237

4 Doctor en Educación. Universidad del AtlánticoColombia. Correo electrónico: roberfigue@hotmail.com.

Orcid: https://orcid.org/0000-0002-0697-5509 ciencias experimentales en el uso del método de Resolución de Problemas en una universidad pública en Colombia. Para tal fin, se hace uso del cuestionario de Representación del Contenido (ReCo) para generar mapa del CPC de seis (6) profesores de ciencias experimentales. Los resultados permitieron identificar las relaciones que se establecen entre los componentes del Conocimiento Pedagógico del Contenido (CPC) en el uso del método de resolución de problemas. Se concluye que cada profesor construye sus propias relaciones a partir de su formación o experiencia. 
PALABRAS CLAVE: ciencias, conocimiento, contenido; estrategia, didáctica

\section{SUMMARY}

This study proposes the characterization of the Pedagogical Knowledge of the Content declared by the university professor of experimental sciences for the use of the Problem Solving method in a public university in Colombia. For this purpose, the Content Representation questionnaire $(\mathrm{ReCo})$ is used to generate a CPC map of six teachers of experimental sciences. The results allowed to identify the relationships that are established between the components of the Pedagogical Content Knowledge (CDC) in the use of the problem-solving method. It is concluded that each teacher builds their own relationships based on their training or experience.

KEYWORDS: science, knowledge, content; strategy, didactics

\section{INTRODUCCIÓN}

El docente universitario de ciencias experimentales debe cumplir un rol importante en el proceso de enseñanza y aprendizaje de sus estudiantes; sus instrucciones deben ser determinante y significativas en el aula, con el solo propósito de generar en ellos una comprensibilidad de los fenómenos naturales y de la relación de éstos con las dinámicas de desenvolvimiento de los jóvenes en la sociedad. Esto quiere decir, que el docente no solo debe tener un amplio conocimiento disciplinar, sino también poseer un conocimiento pedagógicodidáctico, con el fin que los estudiantes logren dilucidar las filigranas que se dan en la intimidad de los fenómenos químicos, físicos, biológicos, y al conocimiento, desarrollo y aplicación de los principios y conceptos científicos en la resolución de problemas en contextos conocidos y no conocidos (Wongsopawiro, 2012; Abell, 2007; King \& Newman, 2000). Es decir, enseñar ciencias desde un método no solo está ligado a los procedimientos relacionados para hacer ciencia sino con los procedimientos para aprender ciencia (Pozo, et al., 1994).

La finalidad de este estudio se basa en generar mapa de Conocimiento Pedagógico del Contenido (CPC), que declara el profesor de ciencias experimentales a nivel universitario, en el uso del método de resolución de problemas en su práctica de enseñanza; en la mirada de contexto y pertinencia interna, es decir, evitar el modelo tradicional, las clases monótonas, tediosas, evaluaciones descontextualizadas y planes de estudios incoherentes.

En consecuencia, se pretende que el docente fortalezca sus prácticas de enseñanzas a través de la exploración de otras opciones, que lo conduzcan a transformar su quehacer formativo, propiciando nuevas acciones, reformando $y$ ajustando las necesidades y alternativas que promuevan el sentido de pertinencia, además, de aportar avances en los indicadores de calidad del programa en mención. Por lo tanto, lo expuesto, facilita el aprendizaje de los conocimientos científicos del maestro en formación e involucra los estudiantes en la comprensión de los contenidos anteriores con los nuevos conocimientos, que encaucen a la abstracción, la modelación, la formulación, la discusión, el trabajo en equipo y en general al aprovechamiento de las capacidades personales de cada estudiante.

\section{MARCO TEÓRICO}

\section{CONOCIMIENTO PEDAGÓGICO DEL CONTENIDO}

El conocimiento pedagógico del contenido lo acuñó Shulman (1986) a mediados de los años ochenta, se refiere al conocimiento que utilizan los maestros para la enseñanza y la comprensión de los alumnos. Está valorado como un concepto epistemológico que combina las bases 
del conocimiento del contenido y la pedagogía en una comprensión de cómo se organizan aspectos particulares de una materia y/o curso, adaptado y representado para la instrucción, incluyendo las formas de representar y formular el tema de una materia.

El CPC con el pasar de los años ha tenido adaptaciones, no obstante, el modelo de CPC más usado por los teóricos en las investigaciones relacionadas con las ciencias ha sido el de Magnusson, Krajcik y Borko (1999), quienes plantean que la característica del CPC es su conceptualización como resultado de una transformación de varios tipos de conocimientos para la enseñanza tales como: Orientación hacia la enseñanza de las ciencias(OECN), conocimientos y creencias acerca del currículum de las ciencias (CUU), conocimientos y creencias acerca de los entendimientos de los estudiantes (CEE), conocimientos y creencias acerca de las estrategias de instrucción para la enseñanza de las ciencias (CIER) y los conocimientos y creencias acerca de la evaluación en ciencias (CCE), colocando así el conocimiento de los profesores en el corazón de la investigación en la educación científica

Existen investigaciones donde se observa la relación entre los componentes planteados por Magnusson, Krajcik y Borko (1999) teniendo en cuenta el grado de frecuencias entre el componente de origen y el de destino como es el caso de Chapoo, Thathong, y Halim (2014), reconocen que los profesores privilegian los componentes relacionados con las orientaciones hacia la enseñanza de las ciencias y el conocimiento de evaluación (González y Rossi, 2015). Por otro lado, Großschedl, Harms, Kleickmann y Glowinski (2015) el curriculum, plantean que el conocimiento del contenido y el conocimiento pedagógico son únicos y separables. Finalmente, Mthethwa-Kunene, Onwu y De Villiers (2015) reconocen que el
CPC es una forma compleja de conocimiento construido por los propios profesores.

Lo anterior lleva a los autores de este estudio a mirar en detalle los hallazgos y buscar acciones que permitan evidenciar elementos relevantes sobre las relaciones de los componentes del CPC.

\section{MÉTODO DE RESOLUCIÓN DE PROBLEMAS}

Para la enseñanza de las ciencias el profesor no es el que aplica instrucciones, sino es un constructivista que piensa y que transforma vidas a través de su acto pedagógico y disciplinar en el aula de clase. Marcelo (1993) establecen que el paradigma del pensamiento del profesor evoluciona hacia un compromiso con los contenidos. Es por ello, que las estrategias didácticas de los profesores son diferentes según la materia que enseñan. Ya que, cada una tiene una naturaleza diferente, lo que implica, que tiene una manera de enseñarla y aprenderla, que se transmiten por los expertos.

Polya (1945) plantea que la resolución de problemas en ciencias está basada en procesos cognitivos que tienen como resultado encontrar una salida a una dificultad, una vía alrededor de un obstáculo, alcanzando un objetivo que no era inmediatamente alcanzable. No obstante, Johnson (1972), define pensamiento como resolución de problemas. Mayer (1910) y Simón (1992) justifican que el pensamiento es lo que sucede cuando una persona resuelve un problema, es decir, produce un comportamiento que mueve al individuo desde un estado inicial a un estado final, o al menos trata de lograr ese cambio. La resolución de problemas es donde se manifiestan las capacidades cognitivas del individuo.

Por esta razón, surgen los procedimientos heurísticos que pueden ser aprendidos, generalizados $y$ transferidos a distintas 
situaciones como los que plantea Dewey (1910); Polya (1945), Mason, Burton y Stacey (1982), De Guzmán (1988), desde su naturaleza, metodología y evaluación, lo que permite que el profesor de ciencias del Programa de la Licenciatura en Ciencias Naturales y Educación Ambiental, potencie el conocimiento didáctico y fortalezcas a través de este proceso las formas de enseñanzas en ciencias. Pues, lo que se busca en la enseñanza de las ciencias es "desarrollar una actitud comprensiva de los problemas globales, que le permita desarrollar pensamiento científico, reflexionando su propia experiencia para contribuir a las transformaciones de una sociedad" (Macedo, Katzkowicz y Quintanilla, 2006, p.12).

\section{METODOLOGÍA}

El estudio se circunscribe en una metodología cualitativa, su función es describir e interpretar la información que se obtiene de la aplicación del cuestionario Representación del Contenido (ReCo), para generar mapa de Conocimiento Pedagógico del Contenido declarado por el profesor universitario, para el uso del método de Resolución de Problemas en educación superior.

El estudio se sustenta desde la concepción constructivismo, los medios de recolección permiten a los autores acceder a datos para ser observados, descritos e interpretados. Su interés no es medir variables de un fenómeno social sino comprenderlo. El foco central es el análisis de la búsqueda de significados (Ruíz, 2009). Y el diseño se basa en el estudio de caso, pues, su abordaje se centra en la recopilación de datos a partir de la experiencia y conocimientos de los profesores del Programa de Licenciatura en Ciencias Naturales y Educación Ambiental sobre CPC en las prácticas de los Profesores. Tales hechos permitirán conocer la manera como los profesores del Programa en mención de la Universidad de Córdoba comprenden y desarrollan el curso asignado en la malla curricular del Programa, en la formación de nuevos licenciados (Carrero, 1998; Carvalho, 2001).

Por otra parte, en este estudio participaron seis (6) docente de ciencias experimentales que trabajan en la Universidad de CórdobaColombia, de los cuales dos (2) trabajan en el área biológica (Doc B-1, Doc B-2), dos (2) en el área de química (Doc Q-1, Doc Q-2) y dos (2) en el área de física (Doc F-1, Doc F-2), con cualificación en estudios de posgrado, sin y con formación pedagógica y con experiencia en el saber disciplinar.

Para el caso del estudio, se opta el cuestionario Representación del Contenido ( $\mathrm{ReCo}$ ) como la técnica que facilita la obtención de los datos, los cuales portan el significado que tienen los sujetos en estudio sobre el CPC (Corbetta, 2003). El cuestionario ReCo fue enviado por correo electrónico a cada profesor participante de la investigación. Cada docente tuvo 20 días para responder el cuestionario.

Posteriormente, a partir de la información del cuestionario ReCo se realizó un análisis de unidades de información a partir de las respuestas de cada profesora teniendo en cuenta las categorías y subcategorías de análisis. Finalmente, un análisis enumerativo entre componentes del CPC (Ravanal \& LópezCorta, 2016) con el propósito de establecer la relación entre ellos y así poder generar los mapas por cada profesor de las distintas áreas disciplinar. Está relación surge a partir de lo declarado por los profesores en el cuestionario ReCo.

Para sistematizar las relaciones de los componentes (Orientación hacia la enseñanza de las ciencias(OECN), conocimientos y creencias acerca del currículum de las ciencias (CUU), conocimientos y creencias acerca de 
los entendimientos de los estudiantes (CEE), conocimientos y creencias acerca de las estrategias de instrucción para la enseñanza de las ciencias (CIER) y los conocimientos y creencias acerca de la evaluación en ciencias (CCE)) y así construir el mapa del CPC de los profesores de ciencias experimentales, se contabilizó el número de vínculos entre el componente de origen y el componente destino. Luego, se calculó la frecuencia entre componentes $y$, finalmente, se representó gráficamente la dirección del vínculo y la frecuencia de las relaciones vinculantes (ver figura 1).

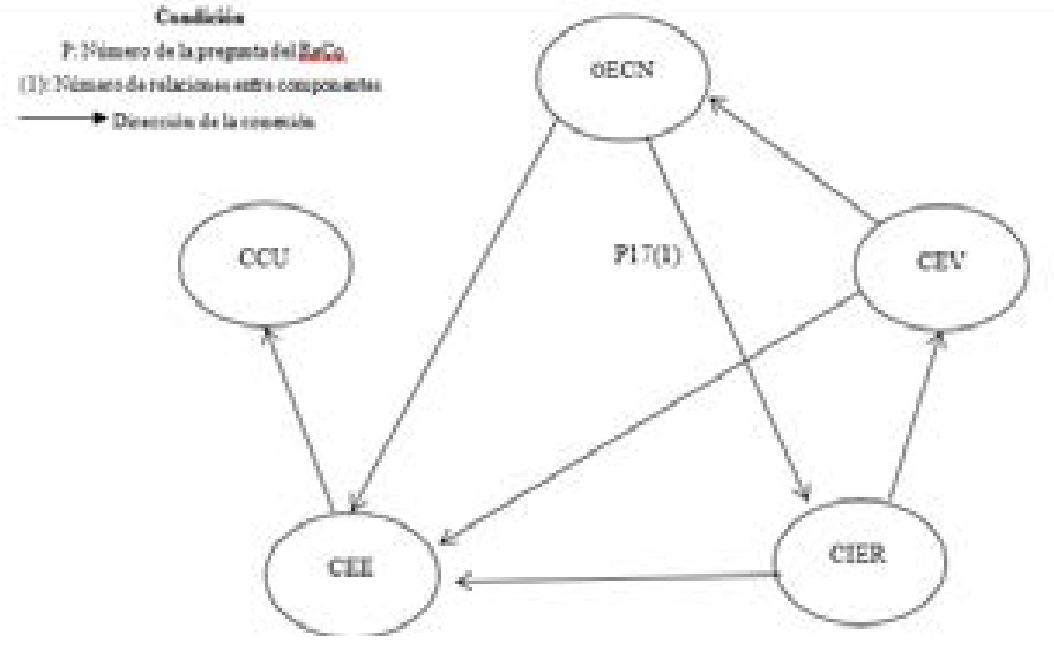

Figura 1. Mapa de la relación entre dominios del CPC. Fuente: Elaboración propia

ANÁLISIS Y DISCUSIÓN DE RESULTADOS

\section{Mapa de relaciones del conocimiento pedagógico del contenido de los docentes del área de Biología.}

El recuento de las relaciones entre los componentes del CPC del Doc B-1, fue de 45. Del total de las relaciones identificada, se observa mayor frecuencia, entre los componentes OECN con un $13 \%$ de relaciones con otros componentes, seguido del CEIR con un $13 \%$ de relaciones con otros componentes y del componente CCE con un $13 \%$ de relaciones con otros componentes. Así mismo, se observa una menor frecuencia en el componente CEE con un $8 \%$ de relaciones con otros componentes y el $53 \%$ del total de las relaciones de cada componente fueron consigo mismo. Ahora bien, del total de las relaciones identificadas, se observa en la figura 2 que el componente de destino que tuvo más frecuencia fue el relacionado CEE y el de menor frecuencia fue el CCU.

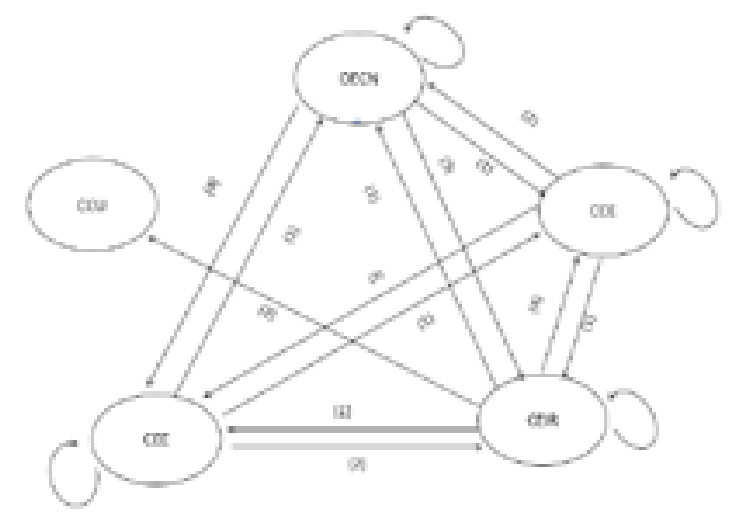

Figura 2. Mapa del CPC del Doc B-1

Fuente: Entrevista aplicada a los profesores de ciencias experimentales del programa de Licenciatura en Ciencias Naturales y Educación Ambiental 
A partir del análisis del cuestionario ReCo se puede afirmar que los procedimientos heurísticos basados en los procesos de pensamiento que desarrolla el Doc B-1 en el aula de clase están influenciados por los planteamientos de algunos autores como Nickerson y Perkins (1985); Polya (1945); Mason et al., (1982) y De Guzmán (1992). En ese orden de idea, en el mapa Doc B-1 se observa que el componente de OECN está centrado en el desarrollo de contenidos biológicos (Valbuena, 2008), competencias específicas y habilidades de pensamiento. En el CCU está relacionado la flexibilidad curricular. En el componente CEE se centra en el rol pasivo y autónomo que asume el estudiante en el aula de clase, y en las ideas previas del estudiante el profesor privilegia las del conocimiento disciplinar. En el componente CEIR se centra en la forma de enseñar a través de clases expositivas, actividades experimentales y las evaluaciones de informes mediadas por las TIC. Y finalmente el CCE está compuesto por una evaluación sumativa y estándar basada en preguntas y que a su vez limita el proceso de aprendizaje de los estudiantes, debido a que evalúa a todos los estudiantes de la misma manera (ver figura 3).

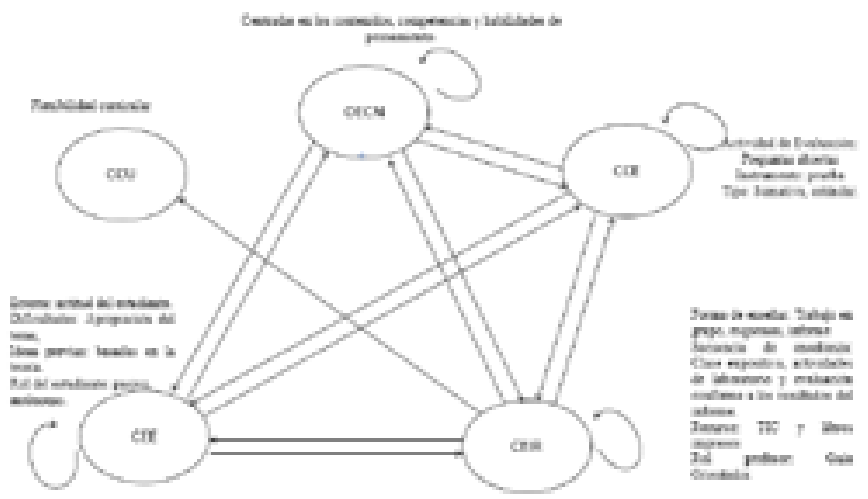

Figura 3. Relación entre dominios del CPC y el modelo de resolución de problemas del Doc B-1. Fuente: Entrevista aplicada a los profesores de ciencias experimentales del programa de Licenciatura en Ciencias Naturales y Educación Ambiental
El recuento de las relaciones entre los componentes del CPC del Doc B-2 fue de 47. Del total de las relaciones identificada, se observa una mayor frecuencia, entre los componentes CEIR con un $23 \%$ de relaciones con otros componentes; el componente CEE con un 17 $\%$ de relaciones con otros componentes, el componente CCE con un $17 \%$ de relaciones con otros componentes y con menor frecuencia. Así mismo, se observa una menor frecuencia en el componente de OECN con un $12 \%$ de relaciones con otros componentes y el $31 \%$ del total de las relaciones de cada componente fueron consigo mismo. Ahora bien, del total de las relaciones identificadas, se observa en la figura 3 que el componente de destino que tuvo más frecuencia fue el OCN y el de menor fue CCE (ver figura 4).

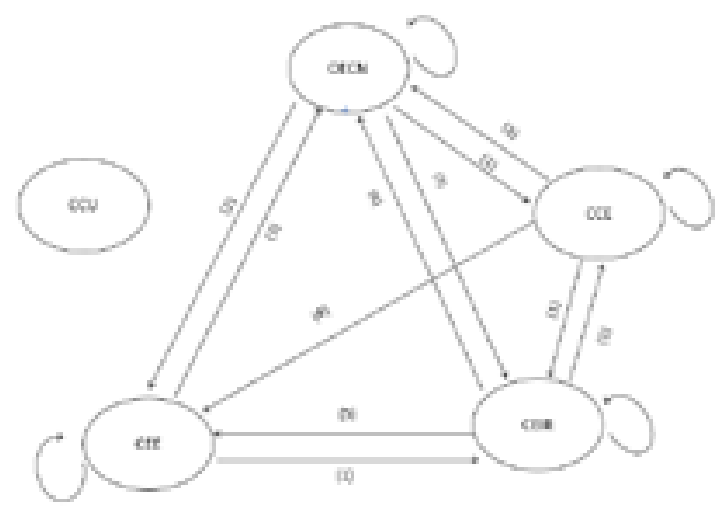

Figura4. Mapa del CPC del Doc B-2

Fuente: Entrevista aplicada a los profesores de ciencias experimentales del programa de Licenciatura en Ciencias Naturales y Educación Ambiental

A partir del análisis del cuestionario ReCo se puede afirmar que los procedimientos heurísticos basados en los procesos de pensamiento que desarrolla el Doc B-2 en el aula de clase están influenciados por los planteamientos como los Nickerson y Perkins (1985); Bacon (1620). En ese orden de idea, en el mapa Doc B-2 se 
observa que el componente de OECN está centrado en el desarrollo de competencias específicas, habilidades de pensamiento (Nickerson y Perkins,1985). En el componente de CEE se centra en la actitud pasiva y miedos que asume el estudiante en el aula de clase, y en cuanto a las ideas previas, el profesor solo privilegia las relacionadas con las de habilidad de pensamiento, dentro las limitaciones se encuentra la comunicación entre estudiantes y docente. En el componente de CEIR se centra en la forma de enseñar a través del método científico apoyado de clases expositivas, actividades mediadas por las TIC - lúdica y las evaluaciones. Y finalmente el CCE basado en la interpretación de los temas aplicando al método científico y la importancia que esto acarrea en cada aspecto de la vida de los estudiantes. Está compuesto por una evaluación sumativa y estándar basada en preguntas y /o situaciones problémicas (ver figura 5).

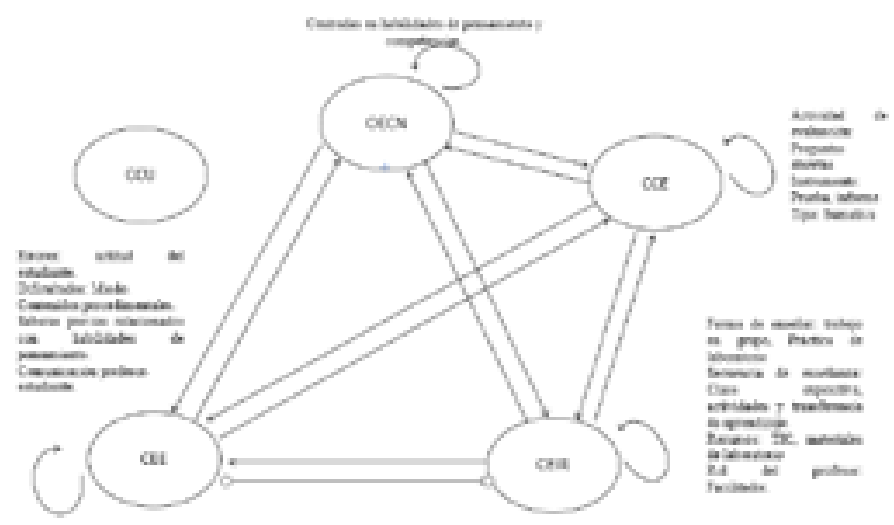

Figura 5. Relación entre dominios del CPC y el modelo de resolución de problemas del Doc B-2.

Fuente: Entrevista aplicada a los profesores de ciencias experimentales del programa de Licenciatura en Ciencias Naturales y Educación Ambiental

\section{Mapa de relaciones del conocimiento pedagógico del contenido de los docentes del área de Química.}

El recuento de las relaciones entre los componentes del CPC del Doc Q-1 fue de
64. Del total de las relaciones identificada, se observa una mayor frecuencia, entre los componentes CEIR $22 \%$ de relaciones con otros componentes, seguido del componente conocimientos y creencias acerca evaluación (CCE) con un $19 \%$ de relaciones con otros componentes, seguido del componente de CEE con un $14 \%$ de relaciones con otros componentes Así mismo, se observa una menor frecuencia en el OECN con un $12 \%$ de relaciones con otros componentes y el 33\% \% del total de las relaciones de cada componente fueron consigo mismo. Ahora bien, del total de las relaciones identificadas, se observa en la figura 6 que el componente de destino que tuvo más frecuencia fue el de OCN y el de menor frecuencia fue CCU.

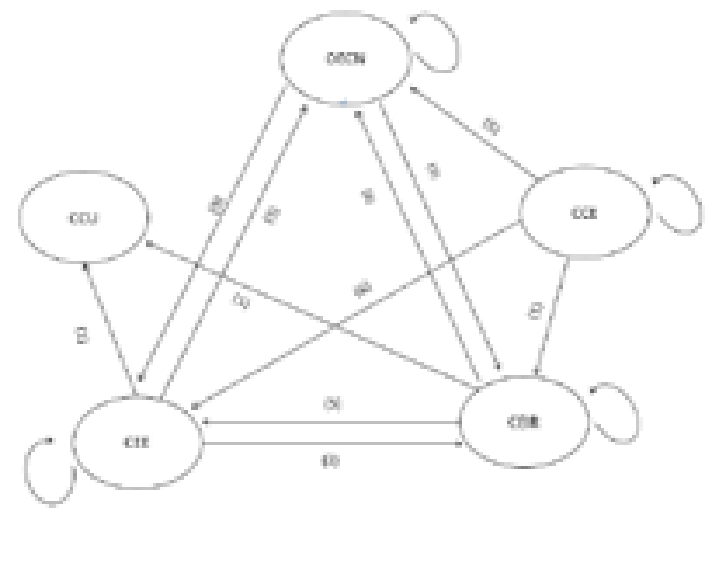

Figura 6. Mapa del CPC del Doc Q-1

Fuente: Entrevista aplicada a los profesores de ciencias experimentales del programa de Licenciatura en Ciencias Naturales y Educación Ambiental

A partir del análisis del cuestionario ReCo se puede afirmar que los procedimientos heurísticos basados en los procesos de pensamiento que desarrolla el Doc Q -1 en el aula de clase están influenciados por los planteamientos de Polya (1945) Mason et al., (1982) y De Guzmán (1992). En ese orden de idea, en el mapa Doc Q-1 se observa que el componente de OECN está centrado en las habilidades de pensamiento 
(Nickerson y Perkins, 1985) y contenidos conceptuales procedimentales. El componente CCU está relacionado de los componentes del plan de curso. El componente CEE se centra en la actitud y dependencia que asume el estudiante en el aula de clase sobre los conocimientos del profesor, dentro de las limitaciones se encuentra la poca apropiación del conocimiento disciplinar que los estudiantes poseen por ello el profesor privilegia en las ideas previas las relacionadas con el saber disciplinar y las relacionadas con operaciones matemáticas. En el CEIR se centra en la forma de enseñar a través del trabajo en grupo. Acompañado de una secuencia didáctica basada en la exploración de conceptos, clases expositivas, actividades mediadas por las TIC y las evaluaciones. $Y$ finalmente el CCE basado en la experimentación con la aplicación de pruebas diagnósticas y sumativas (ver figura 7).

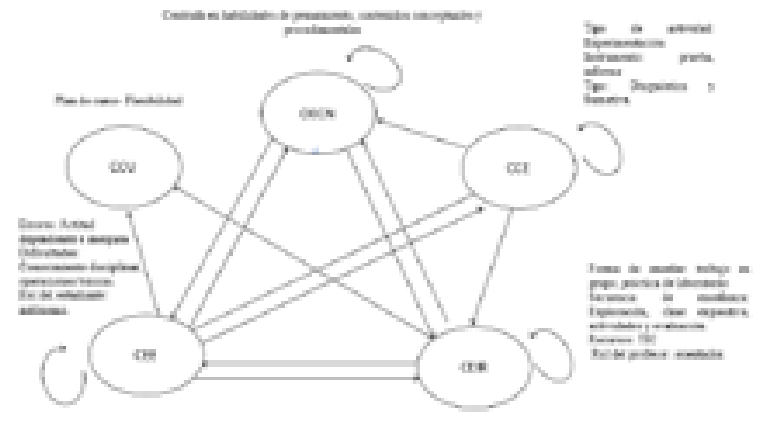

Figura 7. Relación entre dominios del CPC y el modelo de resolución de problemas del Doc Q-1. Fuente: Entrevista aplicada a los profesores de ciencias experimentales del programa de Licenciatura en Ciencias Naturales y Educación Ambiental

El recuento de las relaciones entre los componentes del CPC del Doc Q- 2 fue de 30 . Del total de las relaciones identificada, se observa una mayor frecuencia, entre los componentes de CEIR con un $23 \%$ de relaciones con otros componentes, el componente CCE con un $20 \%$ de relaciones con otros componentes, seguido del OECN con un $16 \%$ de relaciones con otros componentes y por último del componente de CEE con un $14 \%$ de relaciones con otros componentes y el $27 \%$ del total de las relaciones de cada componente fueron consigo mismo. Ahora bien, del total de las relaciones identificadas, se observa en la figura 8 que el componente de destino que tuvo más frecuencia fue el CCU y el componente que no mantuvo convergencia fue el relacionado con CCE.

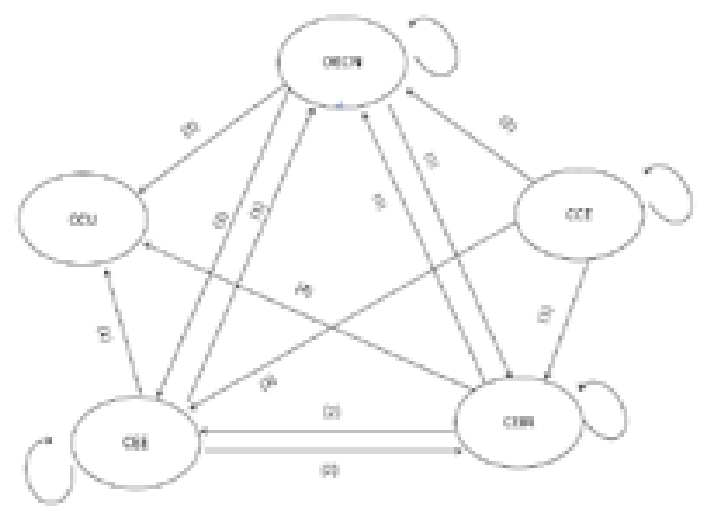

Figura 8. Mapa del CPC del Doc Q-2

Fuente: Entrevista aplicada a los profesores de ciencias experimentales del programa de Licenciatura en Ciencias Naturales y Educación Ambiental

A partir del análisis del cuestionario ReCo se puede afirmar que los procedimientos heurísticos basados en los procesos de pensamiento que desarrolla el Doc Q-2 en el aula de clase están influenciados por los planteamientos de Polya (1945) Mason et al., (1982) y De Guzmán (1992). En ese orden de idea, en el mapa Doc Q-2 se observa que el componente de OECN está centrado en el desarrollo de competencias específicas y habilidades de pensamiento. En el CCU relacionado con la transversalidad con otras áreas de formación. En el componente de CEE se centra los intereses de los estudiantes, en cuanto a las ideas previas el profesor no especifica, sin embargo, menciona que estás deben estar relacionada con las habilidades cognitivas. En el componente de CEIR se centra en la forma de enseñar está dada por 
la relación de la teoría y práctica, en cuanto la secuencia didáctica no especifica. Y finalmente el CCE basado en un trabajo guiado basado en preguntas abiertas, utilizando como instrumento de evaluación prueba sumativa (ver figura 9).

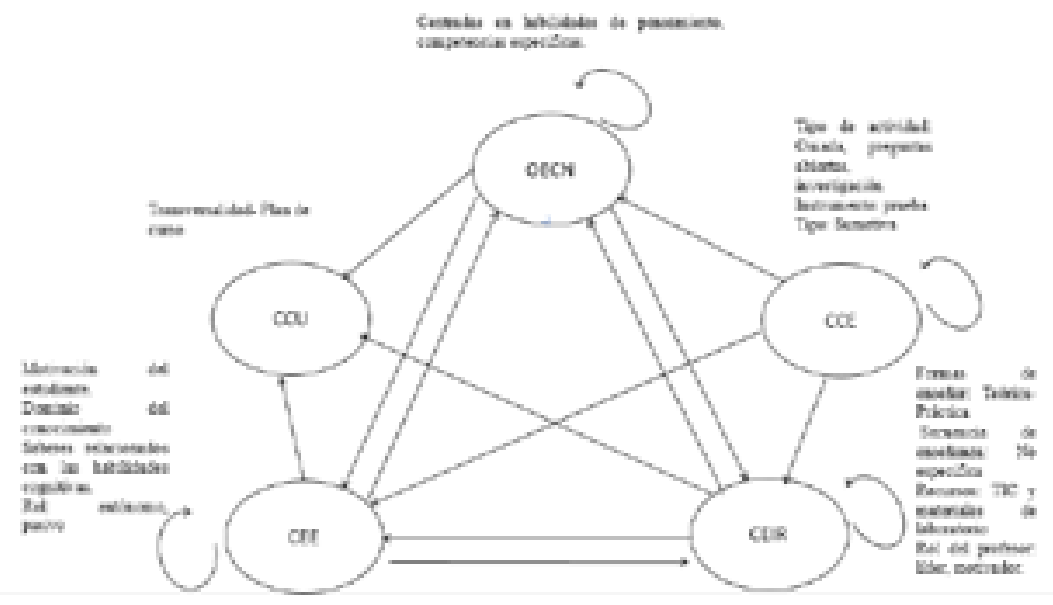

Figura 9. Relación entre dominios del CPC y el modelo de resolución de problemas del Doc Q-2. Fuente: Entrevista aplicada a los profesores de ciencias experimentales del programa de Licenciatura en Ciencias Naturales y Educación Ambiental

\section{Mapa de relaciones del conocimiento pedagógico del contenido de los docentes del área de Físicas}

El recuento de las relaciones entre los componentes del CPC del Doc F - 1 fue de 30. Del total de las relaciones identificada, se observa una mayor frecuencia, entre los componentes de CEIR con un $20 \%$ de relaciones con otros componentes, seguido del OECN con un $17 \%$ de relaciones con otros componentes, el componente de CCE con un $17 \%$ de relaciones con otros componentes, así mismo el componente de CEE con un $10 \%$ de relaciones con otros componentes y el $36 \% \%$ del total de las relaciones de cada componente fueron consigo mismo. Ahora bien, del total de las relaciones identificadas, se observa en la figura 10 que el componente de destino que tuvo más frecuencia OECN y el componente con menor convergencia fue CEIR y CCE.

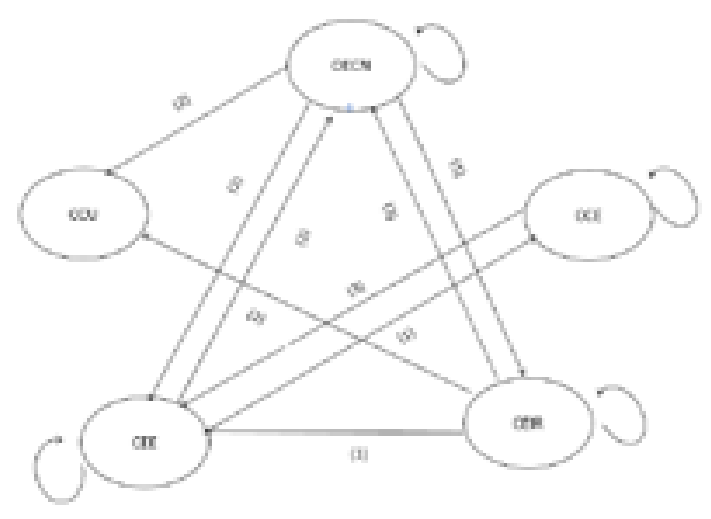

Figura 10. Mapa del CPC del Doc F-1

Fuente: Entrevista aplicada a los profesores de ciencias experimentales del programa de Licenciatura en Ciencias Naturales y Educación Ambiental

A partir del análisis del cuestionario ReCo se puede afirmar que los procedimientos heurísticos basados en los procesos de pensamiento que desarrolla el Doc F-1 en el aula de clase están influenciados por los planteamientos de Polya 
(1945) Mason et al., (1982) y De Guzmán (1992). En ese orden de idea, en el mapa Doc F-1 se observa que el componente de OECN está centrado en el desarrollo de competencias específicas y aprendizaje se concibe como la integración de los contenidos (conceptuales, procedimentales y actitudinales). En el componente de $\mathrm{CCU}$ relacionado con la malla curricular contextualizadas a las necesidades de los estudiantes. En el componente de CEE las dificultades que se presenta es por la poca lectura y profundización, en cuanto a las ideas previas el profesor no especifica sin embargo menciona que estás deben estar relacionada con las operaciones matemáticas, el rol de estudiantes es activo, pero en la mayoría de casos es pasivo, reflexivo y autónomo. En el componente de CEIR se centra en la forma de enseñar e centra en la experimentación, el trabajo guiado y el trabajo en grupo, en cuanto la secuencia didáctica se basa en la explicación teórica que realiza el profesor, las actividades y la trasferencia del aprendizaje. $Y$ finalmente CCE basado en un trabajo guiado basado en preguntas abiertas y talleres, el instrumento para evaluar el aprendizaje es la prueba y el informe, y el tipo de evaluación es sumativa (ver figura 11).

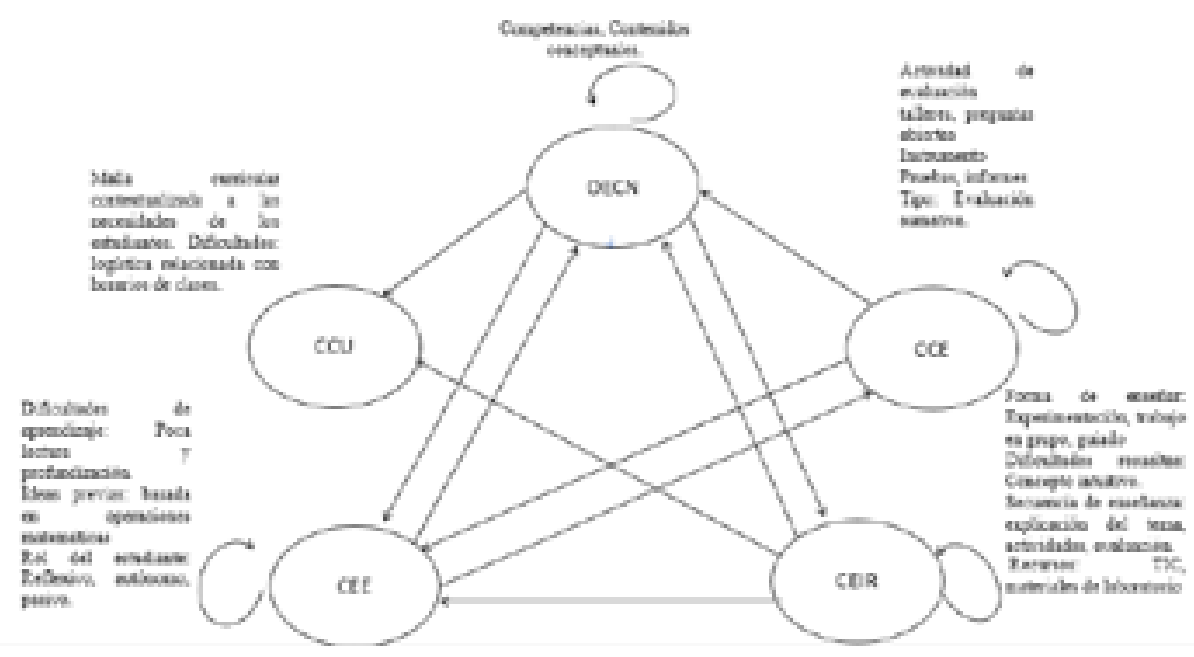

Figura 11. Relación entre dominios del CPC y el modelo de resolución de problemas del Doc F-1. Fuente: Entrevista aplicada a los profesores de ciencias experimentales del programa de Licenciatura en Ciencias Naturales y Educación Ambiental

El recuento de las relaciones entre los componentes del CPC del Doc F- 2 fue de 40. Del total de las relaciones identificada, se observa una mayor frecuencia, entre los componentes de CEIR con un $20 \%$ de relaciones con otros componentes, el componente de CCE con un $20 \%$ de relaciones con otros componentes, seguido del OECN con un $13 \%$ de relaciones con otros componentes y por último del componente CEE con un $13 \%$ de relaciones con otros componentes y el $34 \%$ del total de las relaciones de cada componente fueron consigo mismo. Ahora bien, del total de las relaciones identificadas, se observa en la figura 12 que el componente de destino que tuvo más frecuencia fue el componente de CEE y el componente con menor convergencia fue CCE. 


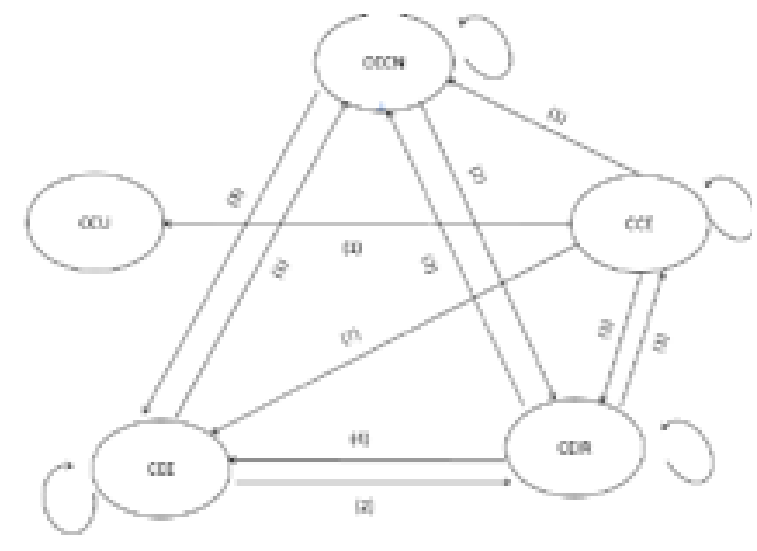

Figura 12. Mapa del CPC del Doc F-2

Fuente: Entrevista aplicada a los profesores de ciencias experimentales del programa de Licenciatura en Ciencias Naturales y Educación Ambiental

A partir del análisis del cuestionario ReCo se puede afirmar que los procedimientos heurísticos basados en los procesos de pensamiento que desarrolla el Doc F-2 en el aula de clase están influenciados por los planteamientos de Polya (1945) Mason et al., (1982) y De Guzmán (1992). En ese orden de idea, en el mapa Doc F-2 se observa que el componente de OECN está centrado en el desarrollo de competencias específicas y habilidades de pensamiento. En el componente de CCU relacionado actualización del plan de curso. En el componente de CEE se centra los intereses de los estudiantes, en cuanto a las ideas previas el profesor no especifica sin embargo menciona que estás deben estar relacionada con el saber especifico y con la naturaleza de la matemática. En el componente de CEIR se centra en la forma de enseñar está dada por la relación de la teoría y práctica, en cuanto la secuencia didáctica está relacionada con la recapitulación de temas, explicación teórica por parte del profesores, actividades y evaluación. $Y$ finalmente el CEE basado en un trabajo guiado basado en preguntas abiertas, utilizando como instrumento de evaluación la prueba el tipo de evaluación formativa (ver figura 13).

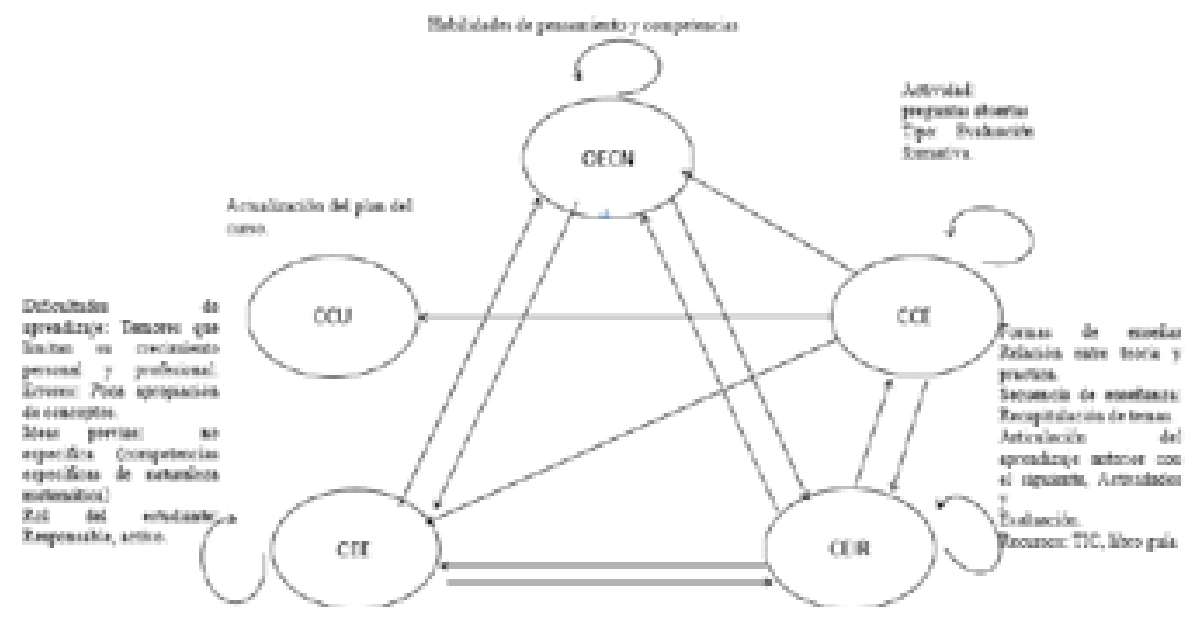

Figura 13. Relación entre dominios del CPC y el modelo de resolución de problemas del Doc F-2. Fuente: Entrevista aplicada a los profesores de ciencias experimentales del programa de Licenciatura en Ciencias Naturales y Educación Ambiental 


\section{CONCLUSIONES}

A partir de la información que se obtiene del diligenciamiento del cuestionario Reco. En los mapas se observó que la relación de los componentes de CPC es diferente en cada caso. Por ejemplo, el Doc Q-1 presento un total de 64 relaciones en tanto que el Doc B-2 es de 47, Doc B-1 es de 45 y Doc F- 2 es de 40, no obstante, las relaciones del Doc Q-2 y Doc F 1 fue de 30 . Este antecedente logra reafirma que el CPC que declaran los profesores de ciencias depende de su formación y experiencia (Ravanal, 2016). Además, se logra identificar y contrastar con los antecedentes que los componentes con menor frecuencia es el relacionado CCU (Großschedl, Harms, Kleickmann y Glowinski, 2015) y CCE. Los componentes con mayores números de relaciones es el componente CEIR y OECN (Chapoo, Thathong, y Halim, 2014). Estos resultados evidencian la influencia que tiene el componente de OECN a la hora de enseñar y de definir la forma de trasmitir sus conocimientos a través de estrategias, uso de recursos. Por otro lado, algo de resaltar en estos resultados es que para los docentes el CEE, está condicionado por el rol que asume el estudiante en el aula de clase y poca apropiación de conocimiento disciplinar y de naturaleza matemática desde el contexto de la escuela.

En ese orden de idea, se evidenció que el conocimiento pedagógico del contenido se encuentro presente en cada profesor participante de la investigación; es decir, cada profesor declara en el aula este conocimiento a partir de su experiencia y formación. A través de la radiografía o mapeo se observó la estructura, características, relaciones entre componentes del CPC, evidenciándose entre componentes relaciones altas y bajas. Lo que conlleva a reflexionar que cada caso es único, por lo que no se puede encasillar a los profesores dentro de un modelo, pues, cada uno de ello, posee su propio modelo a la hora de transmitir sus conocimientos debido al campo disciplinar en el que se desempeñan.

\section{REFERENCIAS BIBLIOGRÁFICAS}

Abell, S. K. (2007). Research on science teacher knowledge. In S. K. Abell \& N. G. Lederman (Eds.), Handbook of research on science education (pp.1105-1149). Mahwah, NJ: Lawrence Erlbaum Associates.

Chapoo, S., Thathong, K., \& Halim, L. (2014). Understanding biology teacher's Pedagogical Content Knowledge for teaching "the nature of organism". Procedia-Social and Behavioral Sciences, 116, 464-471.

Carvalho, J. (2001). La socialización del profesor: Las influencias en el proceso de aprender a enseñar. Tesis doctoral. Universidad de Santiago de Compostela.

Carrero, V. (1998). Análisis cualitativo de datos: Aplicación del estudio de caso de datos Grounded Theory) en el ámbito de la innovación organizacional. Tesis doctoral. Universidad Jaume I (Castellón).

García Franco, A., \& Garritz Ruiz, A. (2006).

Desarrollo de una unidad didáctica: el estudio del enlace químico en el bachillerato. Enseñanza de las Ciencias, 24(1), 111-124.

Garritz, A., \& Trinidad-Velasco, R. (2004). El Conocimiento Pedagógico del Contenido (CPC). Educación química, 15(2), 98-102.

González, N. V., y Rossi, A. M. (2015). Conocimiento pedagógico del contenido para la enseñanza del tema mitosis: un estudio de casos con docentes universitarios de Argentina. Didáctica de las Ciencias Experimentales y Sociales, (29), 215-232.

Großschedl, J., Harms, U., Kleickmann, T., y Glowinski, I. (2015). Preservice biology teachers' professional knowledge: Structure and learning 
opportunities. Journal of Science Teacher Education, 26(3), 291-318.

King, B. M. \& Newman, F. M. (2000). Will teacher learning advance school goals? Phi Delta Kappan, 81(8), 576-580.

Loughran, J., Berry, A. y Mulhall, P. (2006). Understanding and developing science teachers' pedagogical content knowledge. Róterdam: Sense Publishers.

Magnusson, S., Krajcik, J. \& Borko, H. (1999). Naturaleza, fuentes y desarrollo del conocimiento del contenido pedagógico para la enseñanza de las ciencias. En Examinar el conocimiento del contenido pedagógico (pp. 95-132). Springer, Dordrecht.

Macedo, B., Katzkowicz, R. \& Quintanilla, M. (2006), "La educación de los derechos humanos desde una visión naturalizada de la ciencia y su enseñanza: aportes para la formación ciudadana". En: Construyendo ciudadanía a través de la educación científica, Unesco. Disponible en: http://unesdoc.unesco.org/ images/0015/001595/159537S.pdf.

Marcelo, C. (1993). Cómo conocen los profesores la materia que enseñan. Algunas contribuciones de la investigación sobre Conocimiento Pedagógico del Contenido (CPC). En: Montero, L. y Vez, J.M. (Eds.). Las didácticas especificas en la formación del profesorado (pp. 151-185). Santiago: Tórculo.

Mthethwa-Kunene, E., Onwu, G. O., y de Villiers, R. (2015). Exploring biology teachers' Pedagogical Content Knowledge in the teaching of genetics in swaziland science classrooms. International Journal of Science Education, 37(7), 1140-1165.

Pérez-Serrano, M. (2006). Rol docente y pedagogía activa en la formación universitaria. La enseñanza centrada en el aprendizaje del alumno. Adaptación del programa al EEES.

Polya, G., \& Zugazagoitia, J. (1965). Cómo plantear y resolver problemas (No. 04; QA11, P6.). México: Trillas.

Pozo, J.L.; Pérez Echeverría, M.P.; Domínguez, J; Gómez Crespo, M.A.; Postigo, Y. (1994) Solución de problemas Madrid: Santillana/Aula $\mathrm{XXI}$.

Quintanilla, M. (2006). La ciencia en la escuela: un saber fascinante para aprender a "leer el mundo". Revista Pensamiento Educativo, Vol. 39, n², pp. $177-204$.

Quintanilla Gatica, M., \& Ravanal Moreno, L. E. (2009). Racionalidades epistemológicas y didácticas del profesorado de biología en activo sobre la enseñanza y aprendizaje del metabolismo: aportes para el debate de una nueva clase de ciencias (Doctoral dissertation, Universidad Academia de Humanismo Cristiano).

Quintanilla, M. (2014). Las Competencias de Pensamiento Científico desde las 'emociones, sonidos y voces' del aula. Santiago: Editorial Belaterra Ltda.

Ravanal,E., \& López-Corta, F. (2016). Mapa del conocimiento didáctico y modelo didáctico en profesionales del área biológica sobre el contenido de célula. Revista Eureka sobre EnseÃ anza y Divulgación de las Ciencias, 13(3), 725-742. DOI: http://dx.doi.org/10.25267/ Rev_Eureka_ensen_divulg_cienc.2016.v13. i3.15 http://reuredc.uca.es

Ruíz, O (2009). Metodología de la investigación cualitativa. Cuarta Edición. Bilbao. Universidad de Deusto, p.341.

Shulman, L. S. (1986). Those who understand: Knowledge growth in teaching. Educational researcher, 15(2), 4-14.

Shulman, L. S. (2005). Conocimiento y enseñanza: fundamentos de la nueva reforma. 
Revista electrónica. Revista de currículum y formación del profesorado.

Valbuena Ussa, E. O. (2007). El Conocimiento Pedagógico del Contenido (CPC) Biológico: Estudio De Las Concepciones Disciplinares y Didácticas De Futuros Profesores De La Universidad Pedagógica Nacional (Colombia). (Trabajo de grado/tesis doctoral). Universidad Complutense de Madrid. Madrid.

Wongsopawiro, D. S. (2012). Examining science teachers' pedagogical content knowledge in the context of a professional development program. Leiden University Graduate School of Teaching (ICLON), Faculty of Science, Leiden University. 